\title{
Programme de médecine d'urgence hospitalière SSMUS
}

\author{
L. Bernoulli ${ }^{a}$, L. Anselmi ${ }^{b}$
}

Deutsch erschienen

in Nr. 34/2005

\section{a Président SSMUS \\ b Vice-président SSMUS}

Correspondance:

SSMUS

Case postale 1154

CH-8032 Zurich

Tél. 0447991546

Fax 0447991547

E-mail: sgnor.ssmus@bluewin.ch

Internet:

www.sgnor.ch, www.ssmus.ch

\section{Objectif et domaine d'application}

Le programme de médecine d'urgence hospitalière SSMUS définit les critères auxquels doivent répondre les médecins qui occupent une fonction avec des responsabilités médicales dans un service des urgences. Le but est de créer une discipline autonome en médecine d'urgence hospitalière. Le présent programme sert de base, au sens d'une réglementation transitoire, à l'obtention d'une attestation de formation complémentaire en médecine d'urgence hospitalière.

\section{Conditions préalables}

Titre fédéral de spécialiste ou titre de spécialiste étranger reconnu en médecine générale, anesthésiologie, chirurgie, chirurgie orthopédique et traumatologie de l'appareil locomoteur, médecine interne, pédiatrie, chirurgie pédiatrique ou médecine intensive.

\section{Contenu}

3.1 Participation aux cours avec réussite de l'examen dans les domaines suivants:

- Advanced Cardiovascular Life Support selon American Heart Association (ACLSAHA);

- Advanced Trauma Life Support ${ }^{\circledR}\left(\right.$ ATLS $\left.^{\circledR}\right)$;

- Pediatric Advanced Life Support selon American Heart Association (PALS-AHA).

Des cours équivalents peuvent être reconnus par la SSMUS.

3.2 Après l'obtention d'un titre fédéral de spécialiste ou d'un titre de spécialiste étranger reconnu, une activité à plein temps pendant une année ou à 50\% pendant deux ans dans un service des urgences habilité à dispenser la formation de spécialiste.

\section{Dispositions transitoires}

Exigences minimales valables pour cinq ans suite à l'entrée en vigueur du programme:

4.1 Titre fédéral de spécialiste ou titre de spécialiste étranger reconnu en médecine générale, anesthésiologie, chirurgie, chirurgie ortho- pédique et traumatologie de l'appareil locomoteur, médecine interne, pédiatrie, chirurgie pédiatrique ou médecine intensive.

4.2 Activité exercée actuellement en tant que responsable d'un service des urgences ou activité régulière au sein d'un service des urgences dans une discipline apparentée pendant au moins deux ans.

4.3 Participation à un des trois cours avec réussite de l'examen:

- Advanced Cardiovascular Life Support selon American Heart Association (ACLSAHA);

- Advanced Trauma Life Support ${ }^{\circledR}\left(\right.$ ATLS $\left.^{\circledR}\right)$;

- Pediatric Advanced Life Support selon American Heart Association (PALS-AHA). Des cours équivalents peuvent être reconnus par la SSMUS.

\section{Attestation}

L'attestation du programme accompli est du ressort de la Commission pour la formation postgraduée et continue.

\section{Validité et formation continue}

6.1 La validité du programme de médecine d'urgence hospitalière une fois accompli est de cinq ans.

6.2 La validité peut être prolongée de cinq ans si le candidat a effectué 50 heures de formation continue reconnue par la SSMUS conformément à la réglementation de formation continue pour les médecins d'urgence SSMUS.

6.3 L'attestation de la formation continue accomplie est du ressort de la Commission pour la formation postgraduée et continue.

\section{Modifications}

L'assemblée générale décide des modifications du programme.

Approuvé par l'assemblée générale le 22 avril 2005 à Lucerne. 